s. and

$=$

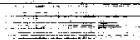

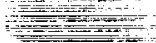

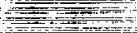

$1+$

$\therefore=-1+2$

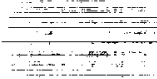

$\frac{=1}{1=}$

a

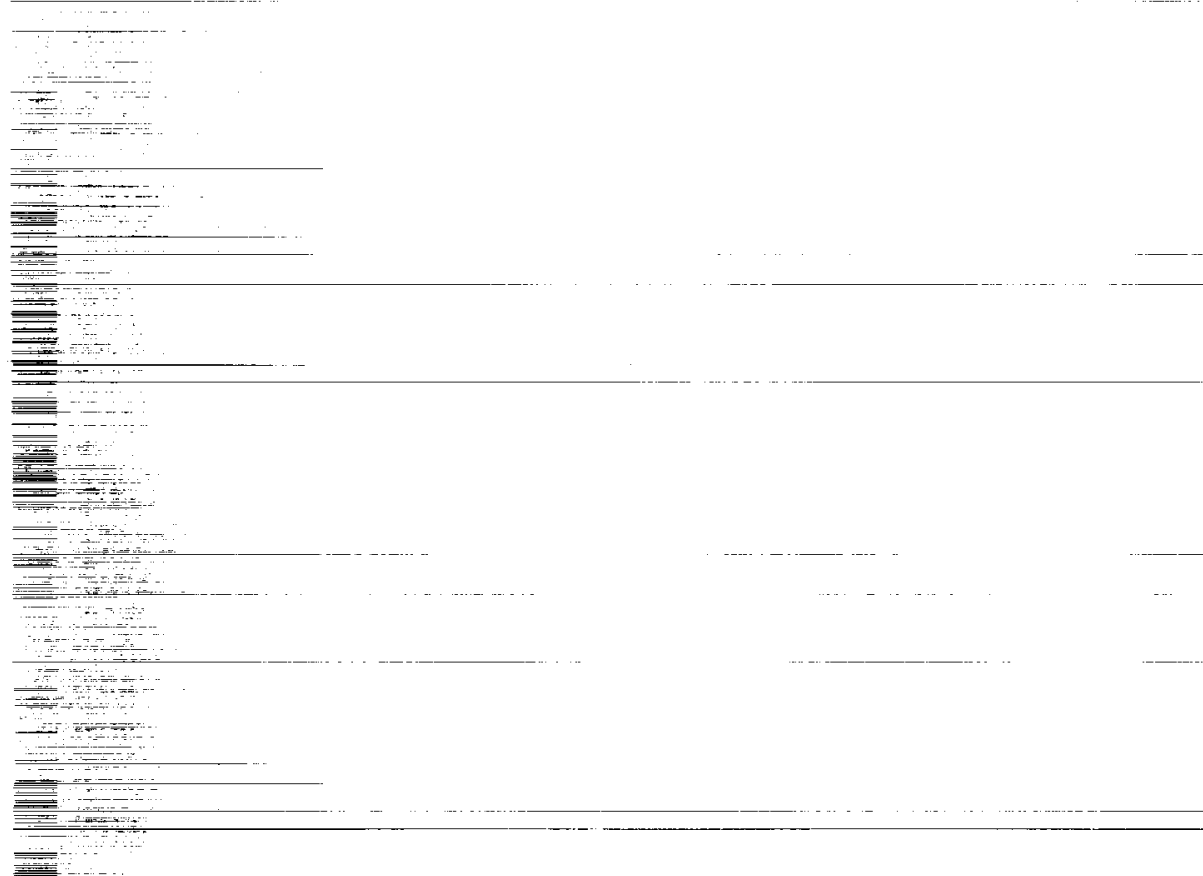

䍿

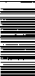

.

군

$=$

…

$\bar{\square}$

$=$

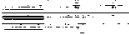

譆

=

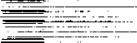

$\equiv$

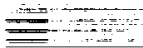

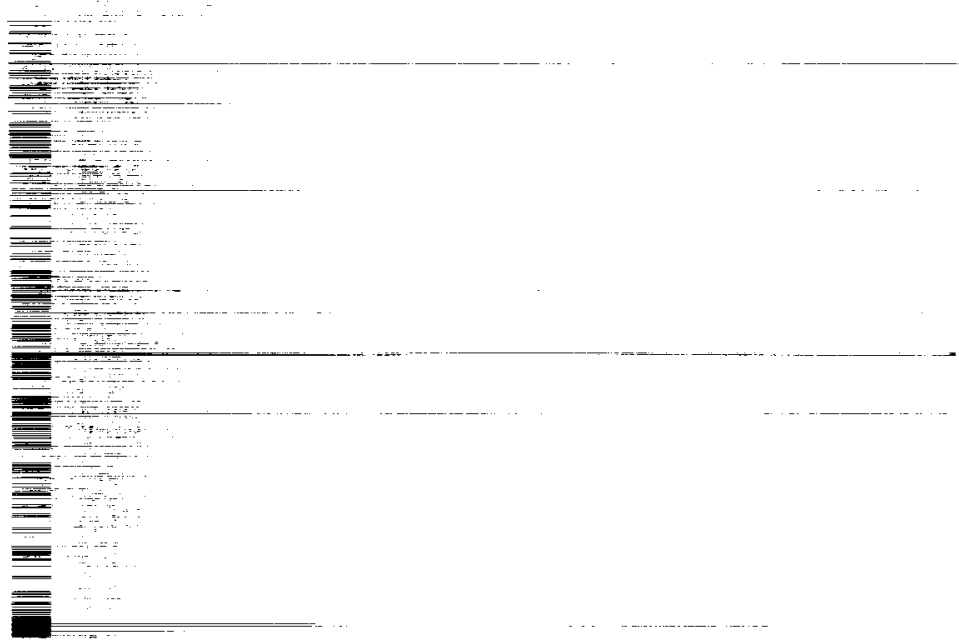


NASA Contractor Report 4460

\section{A Boundary Element Alternating Method for Two-Dimensional Mixed-Mode Fracture Problems}

I. S. Raju and T. Krishnamurthy Analytical Services \& Materials, Inc. Hampton, Virginia

Prepared for

Langley Research Center under Contract NAS1-19317

\section{N/SA}

National Aeronautics and

Space Administration

Office of Management

Scientific and Technical

Information Program 


\title{
A BOUNDARY ELEMENT ALTERNATING METHOD FOR TWO-DIMENSIONAL MIXED-MODE FRACTURE PROBLEMS
}

\author{
I. S. Raju and T. Krishnamurthy \\ Analytical Services \& Materials, Inc. \\ 107 Research Drive, Hampton Va. 23666
}

\begin{abstract}
A boundary-element alternating method, denoted herein as BEAM, is presented for two-dimensional fracture problems. This is an iterative method which alternates between two solutions. An analytical solution for arbitrary polynomial normal and tangential pressure distributions applied to the crack faces of an embedded crack in an infinite plate is used as the fundamental solution in the alternating method. A boundary-element method for an uncracked finite plate is the second solution. For problems of edge cracks a technique of utilizing "finite elements" with BEAM is presented to overcome the inherent singularity in boundary element stress calculation near the boundaries. Several computational aspects that make the algorithm efficient are presented. Finally the BEAM is applied to a variety of two-dimensional crack problems with different configurations and loadings to assess the validity of the method. The method gives accurate stress-intensity factors with minimal computing effort.
\end{abstract}

\section{Introduction}

Stress-intensity factors are fundamental fracture parameters that are needed to design structures against fatigue and fracture failures. In two-dimensional analysis, several methods are available in the literature to calculate the stress-intensity factors of cracked bodies. Several stress-intensity factor compendia [1-4] are also available. Recent research [5-13] revealed the potential of the alternating method to obtain stress-intensity factors in cracked bodies for which solutions are not now readily available. The alternating method developed and employed here is based on an earlier method known as the the Shwartz-Neumann alternating method [5]. The alternating method is an iterative numerical technique that alternates between two solutions to satisfy the required boundary conditions of the problem. One solution is a fundamental and is usually a continuum solution for a cracked infinite plate or solid. The second solution is provided by a numerical analysis such as finite-elements or boundary-elements of the uncracked body subjected to 
the same loading conditions. The method alternates between these two solutions to satisfy the required boundary conditions of the original problem.

The literature contains several papers on the alternating method which use the finite element method to obtain the second solution [6-12]. Recently the boundary element method was used in the alternating method instead of finite-element method [13]. The boundary element method (BEM) is attractive because with this method only the boundaries of the problem need to be modeled and hence, the modeling effort is considerably reduced. The purpose of this paper is to present such a boundary-element alternating method (BEAM) for two-dimensional mixedmode crack problems. The method is thoroughly discussed and several attractive computational features of this method are highlighted. A procedure to combine "finite elements" with BEAM to overcome inherent singularities in stress calculation with BEM is discussed. First, the basic analytical solutions for a crack in an infinite plate subjected to arbitrary normal and tangential pressure distributions over the crack faces are summarized. Second, a brief summary of the boundaryelement method is presented to facilitate the presentation of the BEAM. Next, various computational aspects involved in the BEAM are discussed. Finally, several numerical examples are presented to illustrate the versatility of this method to obtain accurate mixed-mode stress-intensity factors for several cracked configurations.

\section{Analytical solutions}

Use of the alternating method requires analytical solutions for an embedded crack in an infinite plate subjected to tractions on the crack faces. Consider then an infinite plate with a crack of length $2 a$ as shown in Fig. 1. It is assumed that the tractions on the crack faces can be represented using an arbitrary polynomial pressure distribution, $p_{y}$, applied normal to the crack faces and an arbitrary polynomial pressure distribution, $p_{x}$, applied tangential to the crack faces. The normal, $\left(p_{y}\right)$, and tangential, $\left(p_{x}\right)$, pressure distributions applied over the crack faces take the polynomial form, 


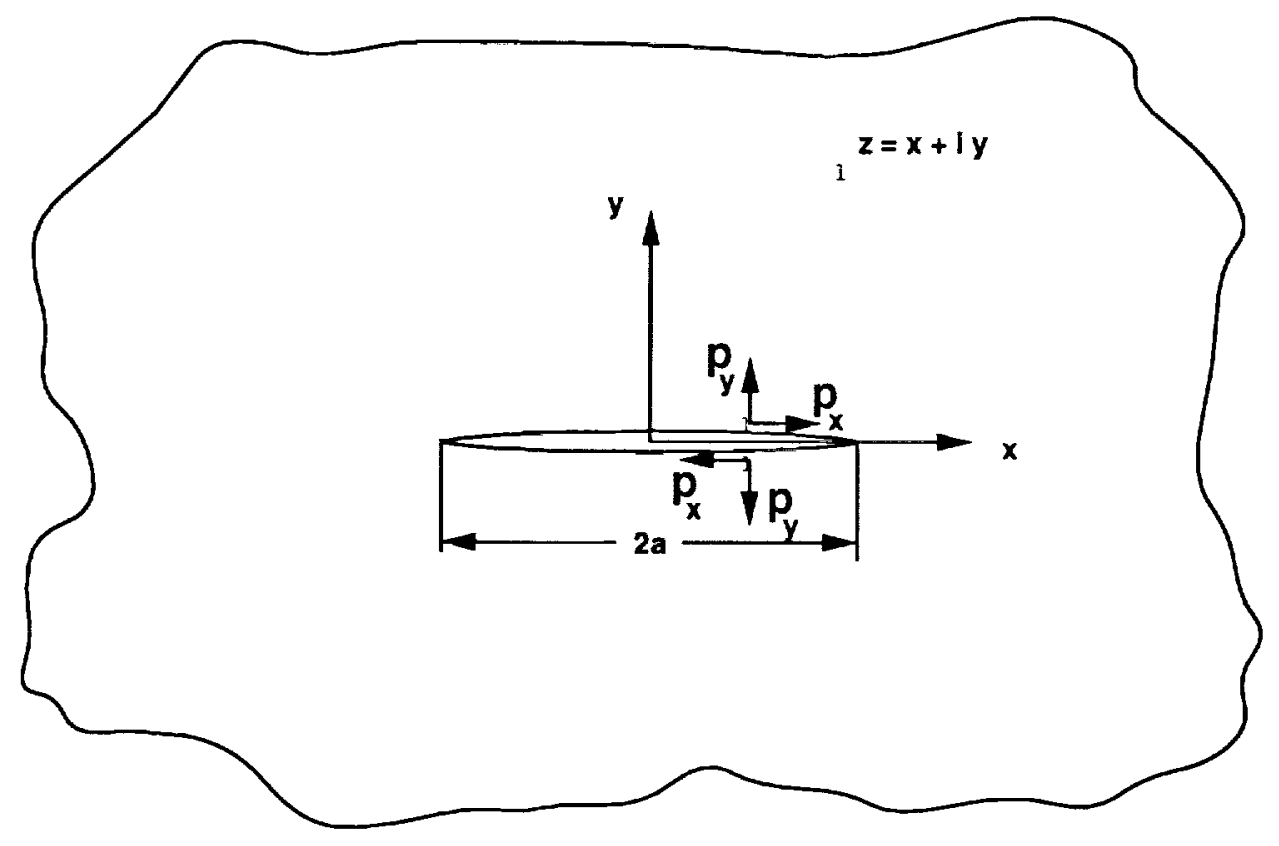

Figure 1. Crack in an Infinite plate subjected to normal and tangential forces

$$
\begin{aligned}
& p_{y}=\sum_{n=0}^{N} A_{n}(x / a)^{n} \\
& p_{x}=\sum_{n=0}^{N} B_{n}(x / a)^{n}
\end{aligned}
$$

where $N$ is the maximum degree of the polynomial functions and $A_{n}$ and $B_{n}$ are arbitrary constants. To obtain the stresses everywhere in the infinite plate due to the above arbitrary pressure distributions, the analytical solutions for a typical polynomial term $(x / a)^{n}$ in Eq. (1) is obtained. The Westergaard stress function for any integer power $n$ can be written as $[10,11]$

$$
\psi(z)= \begin{cases}-(z / a)^{n}+\frac{G_{\infty}^{o}(z)}{\left[a^{n} \sqrt{\left(z^{2}-a^{2}\right)}\right]} & \text { for odd } n \\ -(z / a)^{n}+\frac{G_{\infty}^{e}(z)}{\left[a^{n} \sqrt{\left(z^{2}-a^{2}\right)}\right]} & \text { for even } n\end{cases}
$$


where $z$ is a complex variable, $z=x+i y$ and $i=\sqrt{-1}$

The functions $G_{\infty}^{o}(z)$ and $G_{\infty}^{e}(z)$ are given in reference 10 as

$$
G_{\infty}^{o}(z)=z^{2 m+2} \sum_{k=0}^{m+1} C_{k}\left(\frac{-a^{2}}{z^{2}}\right) \frac{1}{k !}
$$

for odd values of $n$ with $n=2 m+1$

$$
G_{\infty}^{e}(z)=z^{2 m+2} \sum_{k=0}^{m} C_{k}\left(\frac{-a^{2}}{z^{2}}\right) \frac{1}{k !}
$$

and for even values of $n$ with $n=2 m$. In Eqs. (3) and (4) the constants $C_{k}$ are

$$
C_{k}= \begin{cases}1 & \text { for } k=0 \\ \left(\frac{1}{2}\right)\left(\frac{1}{2}-1\right)\left(\frac{1}{2}-2\right) \ldots\left(\frac{1}{2}-k+1\right) & \text { for } k=1,2,3 \ldots\end{cases}
$$

The stress function of Eq. (2) is valid for both normal and tangential distributions of Eq. (1).

The stresses at any general location $z$ in the plate due to normal, $p_{y}$, pressure distribution on the crack faces are [1]

$$
\begin{aligned}
\left(\sigma_{x}\right)_{p_{y}} & =\Re(\psi)-y \Im\left(\psi^{\prime}\right) \\
\left(\sigma_{y}\right)_{p_{y}} & =\Re(\psi)+y \Im\left(\psi^{\prime}\right) \\
\left(\sigma_{x y}\right)_{p_{y}} & =-y \Re\left(\psi^{\prime}\right)
\end{aligned}
$$

Similarly, the stress at any location $z$ in the plate due to tangential, $p_{x}$, pressure distribution, on the crack faces are 


$$
\begin{aligned}
\left(\sigma_{x}\right)_{p_{x}} & =2 \Im(\psi)+y \Re\left(\psi^{\prime}\right) \\
\left(\sigma_{y}\right)_{p_{x}} & =-y \Re\left(\psi^{\prime}\right) \\
\left(\sigma_{x y}\right)_{p_{x}} & =\Re(\psi)-y \Im\left(\psi^{\prime}\right)
\end{aligned}
$$

In equations (6) and (7), $\Re()$ and $\Im()$ denote the real and imaginary parts, respectively, of the function in the parenthesis and

$$
\psi^{\prime}=\frac{d \psi}{d z}
$$

The stress-intensity factor weights, $\left(k_{w}\right)_{n}$, for mode $I$ and mode $I I$ for a typical term $(x / a)^{n}$ in Eq. (1) can be obtained from the stress function as

$$
k_{w}=\sqrt{2 \pi} \lim _{z \rightarrow a}\{\sqrt{z-a} \psi(z)\}
$$

for the crack tip at $x=a$ and

$$
k_{w}=\sqrt{2 \pi} \lim _{z \rightarrow-a}\{(-i) \sqrt{z+a} \psi(z)\}
$$

for crack tip at $x=-a$.

The stress-intensity factors for each of the polynomial terms $(x / a)^{n}$ were computed and are presented in Table 1.

\section{The boundary element method}

In this section, the boundary-element method (BEM) is briefly outlined to facilitate the presentation of the BEAM. In the absence of body forces the integral representation of the displacement at any internal point ' $j$ ' for a two-dimensional domain can be written as [14] 
Table 1 Stress-intensity factor weights for a crack in an infinite plate subjected to arbitrary normal $\left(p_{y}\right)$ and tangential pressure $\left(p_{x}\right)$ distributions of the form $(x / a)^{n}$

\begin{tabular}{lc}
\hline $\mathrm{n}$ & $\left(k_{w}\right)_{n}$ \\
\hline 0 & 1 \\
1 & $\pm 1 / 2$ \\
2 & $1 / 2$ \\
3 & $\pm 3 / 8$ \\
4 & $3 / 8$ \\
5 & $\pm 5 / 16$ \\
6 & $5 / 16$ \\
7 & $\pm 35 / 128$ \\
8 & $35 / 128$ \\
\hline
\end{tabular}

The postive and negative signs in this table refer to the crack tips at $x= \pm a$, respectively. The negative values are meaningful only in the presence of additional forces which prevent crack closure.

$$
u_{l}\left(\xi_{j}\right)+\int_{\Gamma} p_{l k}^{*}\left(x_{j}, \xi_{j}\right) u_{k}\left(x_{j}\right) d \Gamma=\int_{\Gamma} u_{l k}^{*}\left(x_{j}, \xi_{j}\right) p_{k}\left(x_{j}\right) d \Gamma
$$

where $u_{l k}^{*}, p_{l k}^{*}$ are components of displacements and tractions obtained from the fundamental solution of a unit point load in an infinite domain. Specifically, $u_{l k}^{*}\left(x_{j}, \xi_{j}\right)$ is the displacement along the $x_{l}$ - direction at point $x_{j}$, due to a unit point load along the $x_{k}$ - axis at point $\xi_{j}$ in an infinite domain of elastic linear isotropic material. Similarly, $p_{l k}^{*}\left(x_{j}, \xi_{j}\right)$ is the traction along the $x_{l}$ - axis on a line oriented at point $x_{j}$ due to a unit point load along the $x_{k}$ - axis at point $\xi_{j}$ in an infinite domain. The quantity $u_{k}\left(\xi_{j}\right)$ are the displacements at point $\xi_{j}$ and so on. Equation (10) is the well-known Somigliana's identity and it gives the values of the displacements at any internal point in terms of the boundary values $u_{k}, p_{k}$ [14].

In the BEM the boundary (or boundaries) of the domain is discretized into $N_{e}$ number of boundary elements. The displacements $u_{k}$ and tractions $p_{k}$ within each of these elements are assumed to vary in a constant or linear or quadratic manner. The discretized form of Eq. (10) is written as 


$$
u_{k}+\sum_{i=1}^{N_{e}} \int_{\Gamma_{n}} p_{l k}^{*} u_{k} d \Gamma_{n}=\sum_{i=1}^{N_{e}} \int_{\Gamma_{n}} u_{l k}^{*} p_{k} d \Gamma_{n}
$$

A boundary integral expression can be obtained by taking Eq. (11) to the boundary. This equation when applied to different points on the boundary produces a system of equations that are of the form

$$
[\tilde{H}]\{U\}=[\bar{G}]\{T\}
$$

where $\{U\}$ and $\{T\}$ are the displacements and tractions, respectively, at all the nodes on the boundary $\Gamma$. For a mixed boundary value problem, the displacements $\left\{U_{p}\right\}$ are prescribed on some parts of the boundary and tractions $\left\{T_{p}\right\}$ are prescribed on the remaining parts of the boundary. Note that at any point on the boundary either displacements or tractions are known. Equation (12) can be rearranged to group all the unknowns on the left hand side (LHS) and all the known quantities on the right hand side (RHS). This rearrangement leads to

$$
[H]\{X\}=[G]\{F\}
$$

where $\{X\}$ is the column vector of all the unknown displacements and tractions and $\{F\}$ contains the values of the known displacements and prescribed tractions. Note that unlike finite-element method, the matrix $[H]$ in $\mathrm{Eq}$. (13) is unsymmetric and is fully populated. The unknown displacements and tractions can be obtained solving Eq. (13) as

$$
\{X\}=[H]^{-1}[G]\{F\}
$$

Once the nodal displacements and tractions are known, the displacements, $\{u\}$, and stresses, $\{\sigma\}$, at any internal point can be calculated using the Somigliana's identity as, 


$$
\begin{aligned}
& \{u\}=\sum_{j=1}^{N_{e}}\left[D_{1}\right]_{j}\left\{T_{e}\right\}_{j}-\sum_{j=1}^{N_{e}}\left[D_{2}\right]_{j}\left\{U_{e}\right\}_{j} \\
& \{\sigma\}=\sum_{j=1}^{N_{e}}\left[S_{1}\right]_{j}\left\{T_{e}\right\}_{j}-\sum_{i=1}^{N_{e}}\left[S_{2}\right]_{j}\left\{U_{e}\right\}_{j}
\end{aligned}
$$

where $N_{e}$ is the number of boundary elements and $\left\{T_{e}\right\}$ and $\left\{U_{e}\right\}$ are nodal tractions and displacements of the $j^{\text {th }}$ element. The matrices $\left[D_{1}\right],\left[D_{2}\right],\left[S_{1}\right]$ and $\left[S_{2}\right]$ in Eqs. (15) are obtained from integrating the integrals involved in displacement and stress calculations [14]. However, when the internal point approaches the boundary the integrals involved in the stresses and displacements at the internal point become hyper-singular [14] and hence give rise to numerical errors. A procedure that circumvents this difficulty when the internal point approaches the boundary and obtains accurate stresses and displacements is presented later in this paper.

\section{Boundary element alternating method}

The procedure used in BEAM is very similar to that used in the finite-element alternating method in references [6-12] The procedure is illustrated in Fig. 2, and is explained below.

Step 1: Analyze the same configuration and loading as the given problem, but without the crack using the boundary element method. The boundary element solution gives the displacements and tractions of all the nodes on the boundaries of the uncracked body. (Note that the line $y=0$ corresponds to the line on which the crack is present in the original problem.) 


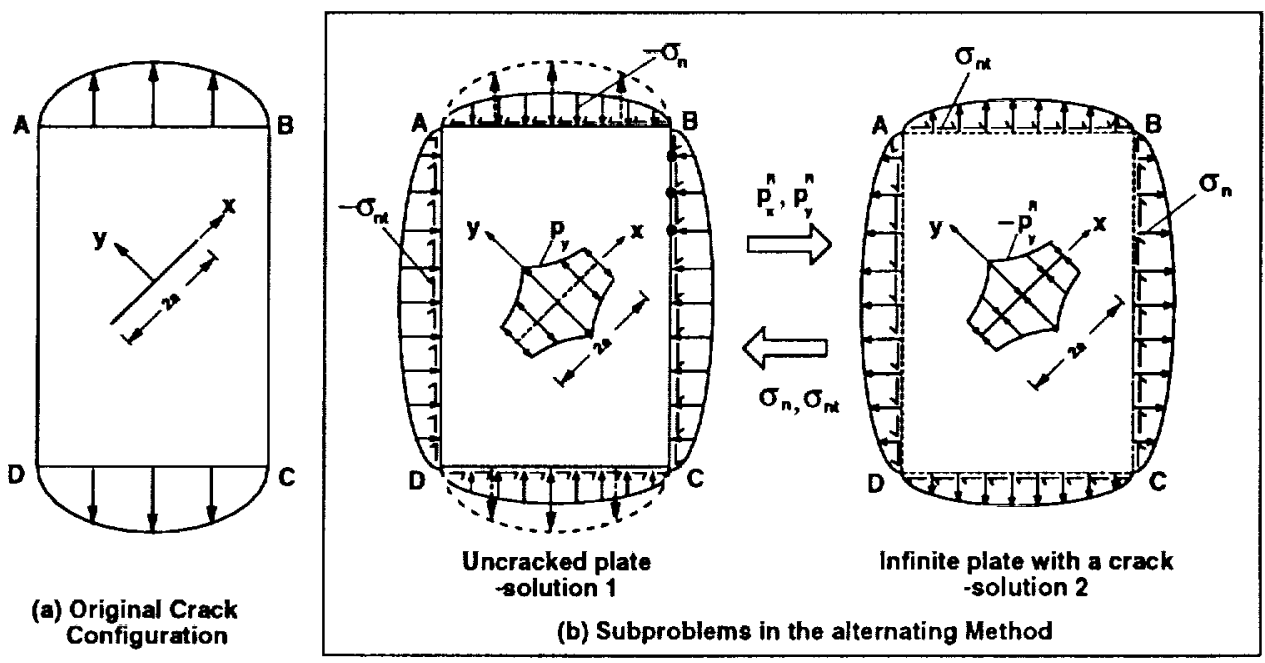

Figure 2. Procedure used in the alternating method

Step 2: Obtain the stresses, $\sigma_{y}$ and $\sigma_{x y}$, on the line $y=0$ for $-a \leq x \leq a$ using Eq. 13. These stresses determine the normal and tangential tractions $p_{y}$ and $p_{x}$, respectively on this line $y=0$.

Step 3: If both the normal and tangential tractions are negligibly small (i.e. smaller than a prescribed tolerance level) stop the algorithm and calculate the sum of the stress-intensity factors computed so far. If either normal or tangential or both tractions are not negligible go to Step 4.

Step 4: For the crack faces to be traction free, the crack face normal tractions $\left(p_{y}\right)$ and tangential tractions $\left(p_{x}\right)$, computed in Step 3 must be removed. (In Fig. 2, for clarity, the tangential tractions on the crack faces are not shown.) Therefore, the negative of crack-face normal tractions (i.e. $p_{y}=-p_{y}^{R}$ ) and crack face tangential tractions (i.e. $p_{x}=-p_{x}^{R}$ ) are applied to the analytical solution. The tractions $p_{y}^{R}$ and $p_{x}^{R}$ are now expressed in polynomial form as

$$
\begin{aligned}
& p_{y}^{R}=\sum_{n=0}^{N} A_{n}(x / a)^{n}=\{P\}^{T}\{A\} \\
& p_{x}^{R}=\sum_{n=0}^{N} B_{n}(x / a)^{n}=\{P\}^{T}\{B\}
\end{aligned}
$$


where

$$
\{P\}^{T}=\left\{1, \quad(x / a), \quad(x / a)^{2}, \cdots \cdots(x / a)^{n}\right\}^{T}
$$

The coefficients $\{A\}$ and $\{B\}$ in Eq. (16) are calculated using a least square procedure [6] by

$$
\begin{aligned}
& \{A\}=[E]^{-1}[D] \\
& \{B\}=[E]^{-1}[C]
\end{aligned}
$$

where

$$
\begin{aligned}
& {[E]=\int_{-a}^{a}\{P\}\{P\}^{T} d x} \\
& {[D]=\int_{-a}^{a}\{P\} p_{y}^{R}(x) d x} \\
& {[C]=\int_{-a}^{a}\{P\} p_{x}^{R}(x) d x}
\end{aligned}
$$

The integrals in Eqs. (18)-(20) can be computed easily by using numerical integration, such as Gaussian quadrature, because the discrete numerical values of $p_{y}^{R}(x)$ and $p_{x}^{R}(x)$ can be calculated at Gaussian points from the boundary element solution.

Step 5: Once the coefficients $\{A\}$ and $\{B\}$ are determined from Eq. (17), equations (9) are used to calculate the stress-intensity factors for the current $j^{t h}$ iteration as

$$
\begin{aligned}
K_{I}^{j} & =\sum_{n=0}^{N}\left(k_{w}\right)_{n} A_{n}^{j} \\
K_{I I}^{j} & =\sum_{n=0}^{N}\left(k_{w}\right)_{n} B_{n}^{j}
\end{aligned}
$$

where $\left(k_{w}\right)_{n}$ are the stress-intensity factor weights given in Table 1 for each of the polynomial functions. 
Step 6: The crack-face normal $p_{y}^{R}$ and tangential $p_{x}^{R}$ tractions obtained in Step 4 create tractions on all the boundaries of the region of interest. The stresses at any point $z=x+i y$, on the boundary can be obtained using Eqs. (2)-(8) for each of the polynomial functions as

$$
\{\sigma\}=[M]\{A\}+[L]\{B\}
$$

where

$$
\{\sigma\}^{T}=\left\{\sigma_{x} \quad \sigma_{y} \quad \sigma_{x y}\right\}
$$

In Eq. (22), $[M]$ and $[L]$ are the stresses at any point $z$ on the boundary due to unit values of each of the polynomial normal and tangential pressure distributions, respectively. Note that the matrices $[M]$ and $[L]$ are available from Eqs. (6) and (7).

The tractions, $T_{x}$ and $T_{y}$, in the $x$ - and $y$-directions, respectively, can be calculated using the stresses on the boundary as,

$$
\{T\}=[q]\{\sigma\}
$$

where

$$
\begin{aligned}
& \{T\}^{T}=\left\{\begin{array}{ll}
T_{x} & T_{y}
\end{array}\right\} \\
& {[q]=\left[\begin{array}{ccc}
n_{x} & n_{y} & 0 \\
0 & n_{y} & n_{x}
\end{array}\right]}
\end{aligned}
$$

In Eq. (25), $n_{x}$ and $n_{y}$ are the direction cosines of the normal to the boundary with respect to $x-$ and $y$-axes, respectively.

Step 7: To satisfy the traction-free boundary conditions on the external boundaries, the tractions created on the these boundaries due to the residual pressures on the crack face in Step 6 need to be removed. Therefore, the negative of these tractions calculated at the nodes in Step 6 are applied to the boundary element model of the uncracked plate. The tractions calculated in Eq. (22) at each node of the model are assembled to form a global vector as 


$$
\{T\}=-\left[\tilde{G}_{n}\right]\{A\}-\left[\tilde{G}_{t}\right]\{B\}
$$

where $\left[\tilde{G}_{n}\right]$ and $\left[\tilde{G}_{t}\right]$ are the assembled matrices obtained using Eqs. (22),(24) and (25) for each node in the model.

The nodal tractions in Eq. (26) along with the original displacement boundary conditions are prescribed as tractions and displacements to the uncracked body. This is the start of the next iteration. This iterative procedure is continued until the crack face tractions in Step 3 are negligibly small. In the converged solution, the mode $I$ and mode $I I$ stress-intensity factors are simply the sum of the stressintensity factors from all the iterations. The convergence criteria were formulated in terms of the integral of the residual pressures on the crack faces and are described in reference 10 . Here, similar convergence criteria are used for both normal and tangential tractions simultaneously.

\section{Some Computational Aspects}

In the boundary element part of the method parabolic straight elements with 3 -nodes were used to model the uncracked boundaries of the body. Several computational aspects of the alternating method which are generic to any numerical method used to analyze the uncracked body are presented in $[6,7,10,12]$. Other computational aspects pertinent to the BEAM are summarized below.

\subsection{Residual Pressure Calculation}

The residual pressures fit over the crack center line (where the crack is present in the original problem) and each iteration requires evaluation of the integrals in Eqs. (18)-(20). To evaluate these integrals the values of stresses at quadrature points are required. These stresses at quadrature points are calculated by using Eq. (15) and assembling contributions from all the boundary elements in the structure. This needs to be performed for each iteration since, the displacements and tractions used in Eq. (15) differ from iteration to iteration. However, considerable computational efficiency can be achieved by a technique described below.

For any given crack length $a$, the coordinates of the quadrature points used in Eqs. (18)-(20) do not vary from iteration to iteration. Hence, before starting the iterative process the matrices $\left[S_{1}\right]$ and $\left[S_{2}\right]$ in Eq. (15) for each of the boundary elements are calculated at the quadrature points. These matrices are then 
assembled to form the global matrices $\left[S_{1 g}^{*}\right]$ and $\left[S_{2 g}^{*}\right]$ for each of the quadrature points. Then the stresses at a quadrature point can be evaluated from,

$$
\{\sigma\}=\left[S_{1 g^{*}}{ }^{*}\{U\}_{k}-\left[S_{2 g}^{*}\right]\{T\}_{k}\right.
$$

where $\{U\}_{k}$ and $\{T\}_{k}$ are the global nodal displacement and traction vectors for the $k^{\text {th }}$ iteration. The stresses at all the quadrature points for each iteration are obtained by simple multiplication involved in Eq. (27). This procedure, however, requires storage of the matrices $\left[S_{1 g}^{*}\right]$ and $\left[S_{2 g}^{*}\right]$ at each of the quadrature points.

\subsection{Calculation Of Nodal Tractions And Displacements}

The unknown nodal tractions and displacements for any $k^{\text {th }}$ iteration are obtained by combining Eqs. (13) and (26) as

$$
\{X\}_{k}=[Q]\{A\}_{k}+[R]\{B\}_{k}
$$

where

$$
\begin{aligned}
& {[Q]=-[H]^{-1}[G]\left[G_{n}\right]} \\
& {[R]=-[H]^{-1}[G]\left[G_{t}\right]}
\end{aligned}
$$

and $\left[G_{n}\right]$ and $\left[G_{t}\right]$ are formed from the matrices $\left[\tilde{G}_{n}\right]$ and $\left[\tilde{G}_{t}\right]$ of Eq. (26) and the prescribed displacement boundary conditions.

As seen from Eq. (28), the unknowns $\{X\}_{k}$, for the $k^{\text {th }}$ iteration depend only on the polynomial coefficients $\{A\}_{k}$ and $\{B\}_{k}$ and the matrices $[Q]$ and $[R]$. The matrices $[H],[G],\left[G_{n}\right],\left[G_{t}\right]$ and hence $[Q]$ and $[R]$ do not vary in the iterative process. Therefore, the assembled LHS matrix $[H]$ needs to inverted only once and the matrices $[Q]$ and $[R]$ need to be computed only once using Eq. (29). This can be performed conveniently before the start of the iterative process. For each iteration, after the residual pressure fit is made to evaluate $\{A\}_{k}$ and $\{B\}_{k}$, the unknowns $\{X\}_{k}$ are immediately available from $\mathrm{Eq}$. (28) by simple matrix multiplications. 


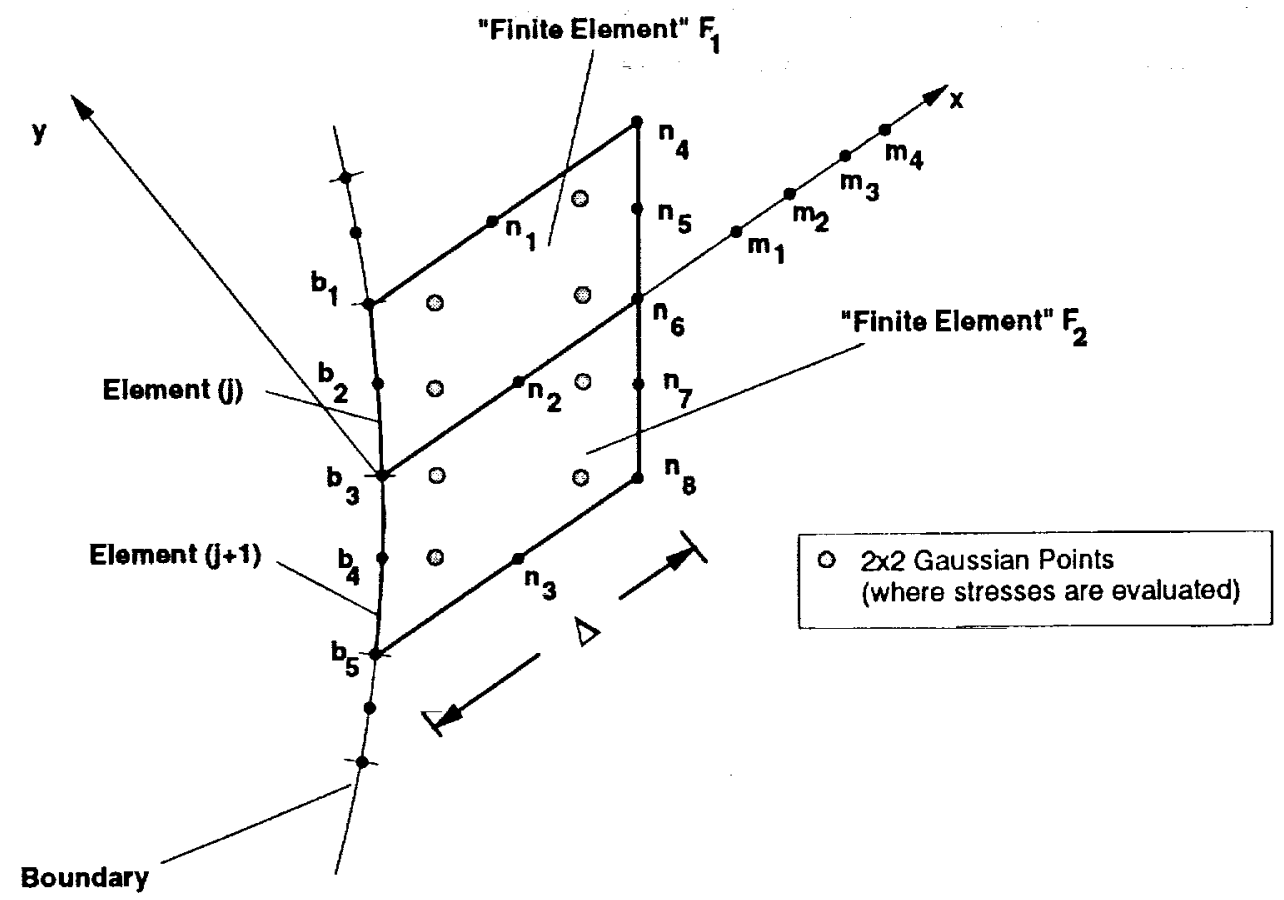

Figure 3. "Finite elements" near the edge for accurate stresses

\subsection{Stresses and Displacements Near Boundary Points}

For edge crack problems, the residual polynomial pressures over the crack line requires estimation of stresses from the BEM portion of the analysis at points very near the boundary where the crack emanates. As mentioned earlier, as the internal point approaches the boundary, the integrals involved in Eq. (15) for displacements and stresses at internal points become hyper-singular [14] and because of this singularity the stresses and displacements calculated very near the boundary, in general, are inaccurate. This can be overcome by integrating the integrals in Eq. (15) in closed form. However, this is not feasible for higher order boundary elements. Therefore, when the internal points are very close to the boundaries special procedures need to adopted to obtain accurate stresses. One such procedure is described below.

The displacements calculated near the boundary points are more accurate than the stresses calculated at the same point. This is because, the singularity in Eq. (15) for displacement calculations near the boundary is one order less than the singularity in stress calculation. This fact is utilized in an extrpolation procedure. In this procedure two "finite elements" are constructed as shown in Fig. 3, near the 
point $b_{3}$ on the edge from which the crack emanates. In Fig. 3, the crack is along the line $b_{3} x$. The nodes $b_{1}, b_{2}, b_{3}, b_{4}, b_{5}$ are the nodes in the boundary element model and $j$ and $(j+1)$ are the elements near the "crack line" of the uncracked body. The two "finite elements" ( $F_{1}$ and $F_{2}$ in Fig. 3$)$ are constructed such that they (a) are like 8-node parabolic finite elements, (b) are physically located on either side of the crack line and share a common side $\left(b_{3} n_{2} n_{6}\right)$ - the crack line, (c) share nodes that belong to the boundary element idealization of the uncracked body $\left(b_{1}, b_{2}, b_{3}\right.$ for the element $F_{1}$ and $b_{3}, b_{4}, b_{5}$ for element $\left.F_{2}\right)$ and (d) penetrate a distance $\Delta$ into the uncracked body.

The displacement at the nodes $n_{1}, n_{2}, \ldots, n_{8}$ in the two "finite elements" can be computed by using Somigliana's identity. The displacements at the boundary $b_{1}, b_{2}, \ldots, b_{5}$ are known from the boundary element solution of the uncracked body. Thus the displacements at all the 8-nodes of each of the finite elements $F_{1}$ and $F_{2}$ are known. Following well known isoparametric finite element procedures the stresses at the $2 \times 2$ Gaussian points are calculated in each of the finite elements. The $2 \times 2$ Gaussian stresses are then extrapolated to the crack center line $[10,15,16]$. In general, the crack line stresses thus obtained from the top element $F_{1}$ are not identical to those obtained from the bottom element $F_{2}$. Therefore, the extrapolated stresses from $F_{1}$ and $F_{2}$ are averaged and are used in the residual fit.

For points on the crack line whose distances from the edge are greater than $\Delta$ (like points $m_{1}, m_{2}, \ldots m_{4}$ in Fig. 3), the stresses can be calculted using the Somigliana's identity, since the boundary element stresses are accurate at these points.

In this procedure, the critical parameter is distance $\Delta$, the length of the "finite elements". Numerical experimentation suggests that the length $\Delta$ should be equal to or up to 1.5 times the length of the smallest element near the edge in the boundary element model of the uncracked body. This procedure appears to demand extra computations. However, the extra computing is well worth the effort because the stresses computed by this procedure are much more accurate than those obtained by conventional boundary element procedures.

\subsection{Consistent Tractions due to Residual Pressure Distribution}

In step 6 of the BEAM, the tractions on all the boundaries due to the residual pressure distributions $p_{x}$ and $p_{y}$ on the crack faces are determined. If the variation of the tractions over an element is higher than a quadratic, then directly using 
the nodal values of the tractions computed from Eq. (24) in the boundary element equations will be inconsistent. This is because the traction computed at any point, $I$, on the boundary element $j$ (see figure 4) using Eq. (24), $T(s)$ is not the same as the traction computed $T_{I}$ at the same point by using

$$
T_{I}=\Phi_{1} T_{1}+\Phi_{2} T_{2}+\Phi_{3} T_{3}
$$

where $T_{1}, T_{2}, T_{3}$ are the nodal tractions calculated by Eq. (24) and $\Phi_{1}, \Phi_{2}, \Phi_{3}$ are the quadratic shape functions of element $j$. The error $E(s)$, at any point $s$ on the $j^{\text {th }}$ boundary element is

$$
\begin{aligned}
E(s) & =T_{I}-T(s) \\
& =\{\Phi\}_{j}^{T}\{T\}_{j}-T(s)
\end{aligned}
$$

where $\{\Phi\}_{j}^{T}=\left\{\begin{array}{lll}\Phi_{1} & \Phi_{2} & \Phi_{3}\end{array}\right\}_{j}^{T}$ and $\{T\}_{j}^{T}=\left\{\begin{array}{lll}T_{1} & T_{2} & T_{3}\end{array}\right\}_{j}^{T}$

The tractions $\{T\}_{j}$ that minimize the error $E(s)$ can be calculated by using a least square procedure as

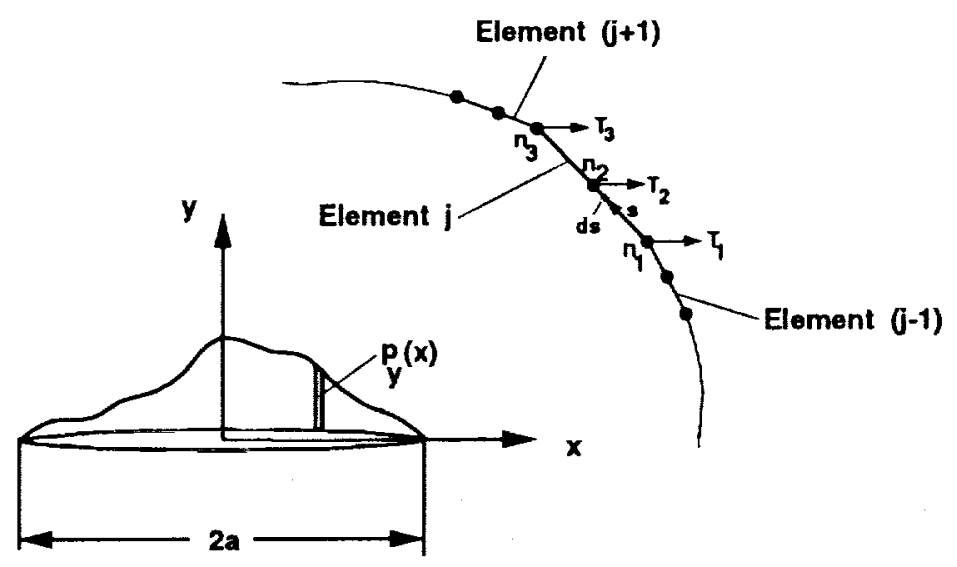

Figure 4. Tractions on element $j$ on the boundary due to crack face pressure loading 


$$
\frac{\partial}{\partial\{T\}_{j}^{T}}\left\{\int_{0}^{l_{j}} E^{2}(s) d s\right\}=0
$$

or

$$
\left(\int_{0}^{l_{j}}\{\Phi\}\{\Phi\}^{T} d s\right)\{T\}_{j}=\int_{0}^{l_{j}}\{\Phi\}^{T} T(s) d s
$$

where $l_{j}$ is the length of the $\mathrm{m} j^{t h}$ element. The Eq. (33) can be concisely written as

$$
\{T\}_{j}=[W]^{-1}\left(\int_{0}^{l_{j}}\{\Phi\} T(s) d s\right)
$$

The integrals involved in Eq. (34) can be carried out numerically because discrete values of $T(s)$ on the elements are available. The matrix $[W]^{-1}$ can be written down explicitly for a 3 -node quadratic boundary element as

$$
[W]^{-1}=\frac{1}{l_{j}}\left[\begin{array}{ccc}
9 & -3 / 2 & 3 \\
-3 / 2 & 9 / 4 & -3 / 2 \\
3 & -3 / 2 & 9
\end{array}\right]
$$

\subsection{Edge Cracks}

The analytical solution used in BEAM is for an embedded crack in an infinite plate having two crack tips. But in edge cracks problems, only one crack tip exists. Hence to use BEAM for edge crack problems, a fictitious crack tip needs to be defined. The fictitious crack tip is usually assumed to be at $x=-a$. While for $0 \leq x \leq a$, the residual pressures are computed from the boundary element solution, the residual pressures on the fictitious part of the crack $-a \leq x \leq 0$ need to be defined. In this paper, a simple constant pressure distribution was used over the fictitious part as discussed in reference 12 . 


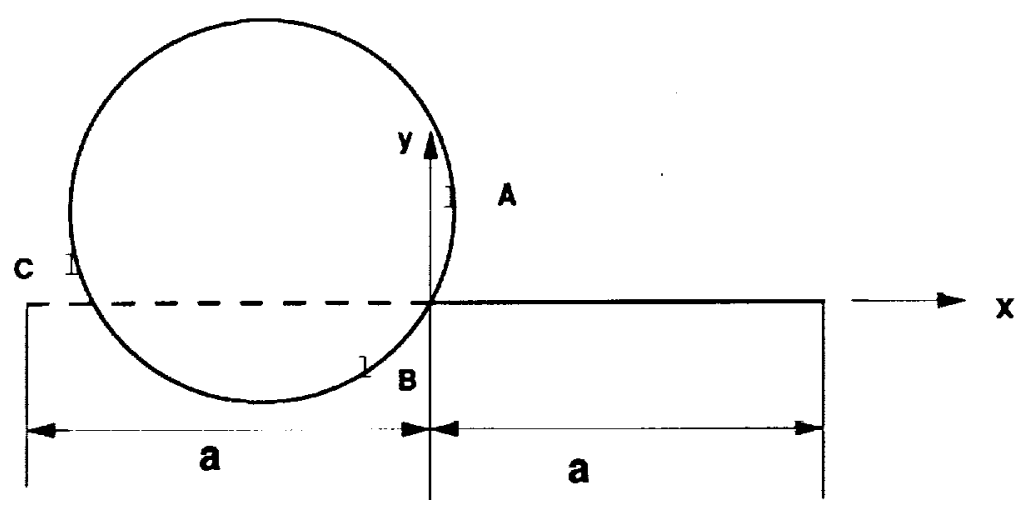

(a) Without shift

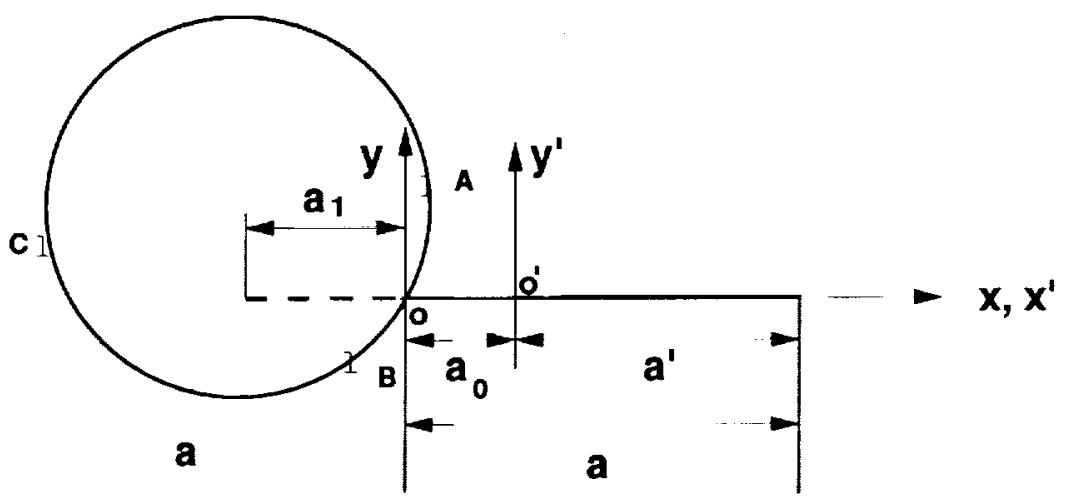

(b) with shift

Figure 5. Orgin shifting to account for the position of the fictious crack tip for edge cracks from internal boundarles

If the crack length is large, the fictitious crack tip can penetrate the boundary opposite the point of origination of the actual crack. One such example of a long edge crack from a circular hole is illustrated in Fig. 5(a). There are two difficulties associated with long edge cracks and penetration of the fictitious part of the crack. First, pressure fitting needs to be carried out on the part of the fictitious crack that is in the penetrated region. This requires complicated boundary element idealization and the construction of "finite elements" at two places on the boundary. The second difficulty pertains to the use of the same boundary element model for all crack lengths. Although only the uncracked body is modeled, the boundary element idealization is usually made finer in the region of the crack and coarse elsewhere. For example, region $A B$ in Fig. 5(a) is usually a fine mesh region and $B C A$ is a coarse mesh region. When the cracks are short the fictitious crack tip,the one at $(-a, 0)$, is closer to the fine mesh region, boundary 
$A B$ in Fig. 5(a). In contrast, when the crack is long, the fictitious crack tip is near the region where the mesh is coarse, like boundary $B C$. Because of the square root singularity at the crack tips, for larger crack lengths the fictitious crack tip creates large tractions on the elements in the coarse mesh region. The iterative process may not be able to erase these tractions created in the elements of the coarse region. In such situations, the iterative process either oscillates or diverges. The current algorithm can be modified to make it more robust and allow the same boundary element model to be used for all crack lengths. The modification is to shift the origin such that the fictitious crack tip is positioned in such a way that its influence is felt most in the region of the fine mesh. Figure 5(b) shows such an origin shift by an amount $a_{0}$ so that the fictitious crack tip is close to the fine mesh region. This method is termed herein, the origin-shifting method. Details of the origin shifting method can also be found in reference [12].

These modifications to the BEAM facilitate the analysis of several crack lengths in a single computer run and require only one inversion of the system matrix in Eq. (14). Furthermore, two different coordinate systems are conveneint for the uncracked body analysis and the analytical solution. A global coordinate system is usually convenient for the uncracked body analysis, while a local (crack) coordinate system is convenient for the analytical part of the method. AppendixA present the transformations that are necessary to be able use two different coordinate systems.

\section{Results and discussion}

To evaluate the effectiveness of the BEAM in mixed-mode problems, the method is applied to several cracked configurations and loadings for which accurate solutions are available in the literature. Both embedded and edge cracks in plates with and without stress concentrations (due to notches and holes) were analyzed. The normalized stress intensity factors are compared with those from the literature [4] for each of these problems analyzed. The percent difference in the tables presented in this paper is defined as

$$
\text { percent difference }=\frac{\mid \text { BEAM value-Reference value } \mid}{\text { Reference value }}
$$

In all the problems analyzed, a plane strain state was assumed with a Poisson's ratio of 0.3 . 
Experience with FEAM [12] showed that the degree of polynomial of value $N=5$ yields accurate stress-intensity factors. Hence $N=5$ was used for all the problems presented in this paper. Several crack lengths were analyzed with a single boundary element model for the uncracked body in a single computer run. All the boundary element idealizations were performed using straight 3-noded quadratic elements. When the prescribed tractions at a boundary point are discontinuous (such as a corner), double nodes were used near that point.

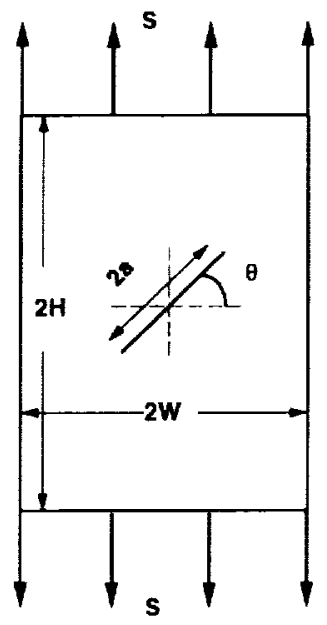

(a) Remote uniform tension loading

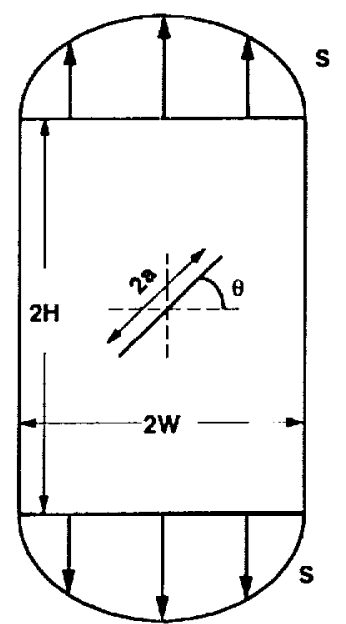

(b) Remote parabolic loading

Figure 6: Embedded slant crack problems analyzed 


\subsection{Embedded Slant Crack Plate}

Remote Uniform Tensile Loading - An embedded slant crack specimen with remote uniform tensile stress is shown in Fig. 6(a). The plate was modeled with 10 quadratic boundary elements and had 24 nodes. The sides $X= \pm W$ were modeled with four equal length elements and the sides $Y= \pm H$ were modeled with one element. Two crack inclination angles $\left(\theta=45^{\circ}\right.$ and $\left.75^{\circ}\right)$ were considered. The boundary element models used are shown in Fig. 7. All the external boundaries, $A B, B C, C D$ and $D A$, in Fig. 7 were made stress free in the iterative part of the algorithm. Eight crack lengths with $(a / W)$ ratios varying from 0.1 to 0.8 were analyzed in a single computer run. Stress-intensity factors were calculated for all crack lengths at both crack tips $(x= \pm a)$. The stress-intensity factors at both the crack tips, as expected, were computed to be identical (to machine accuracy). Tables 2 and 3 present the normalized stress-intensity factors obtained for $\theta=45^{\circ}$ and $75^{\circ}$ respectively. Reference results from the literature are included in these tables for comparison. Excellent agreement is obtained for all crack lengths between the two sets of results even with a very coarse boundary element idealization. The maximum difference is about two percent for both crack inclination angles. Three to five iterations were needed to achieve converged solutions.

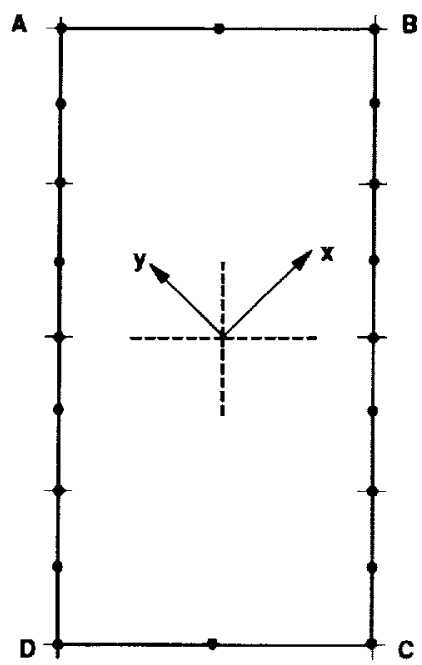

Figure 7. Boundary element model for slant embedded crack problems 
Table 2 Comparison of normalized stress-intensity factors, $F_{I}$ and $F_{I I}$, for embedded slant cracks in a rectangular plate subjected to remote uniform tensile stress. (Fig. $6(\mathrm{a}),(H / W)=2.0, \theta=45^{\circ}$ )

\begin{tabular}{cccccccc}
\hline & \multicolumn{3}{c}{$F_{I}=K_{I} /[S \sqrt{\pi a}]$} & & \multicolumn{3}{c}{$F_{I I}=K_{I I} /[S \sqrt{\pi a}]$} \\
\cline { 2 - 4 }$(a / W)$ & $\begin{array}{c}\text { BEAM } \\
\text { value }\end{array}$ & $\begin{array}{c}\text { Reference } \\
\text { value }\end{array}$ & $\begin{array}{c}\text { Percent } \\
\text { difference }\end{array}$ & $\begin{array}{c}\text { BEAM } \\
\text { value }\end{array}$ & $\begin{array}{c}\text { Reference } \\
\text { value }\end{array}$ & $\begin{array}{c}\text { Percent } \\
\text { difference }\end{array}$ \\
\hline 0.1 & 0.505 & 0.505 & 0.0 & 0.502 & 0.502 & 0.0 \\
0.2 & 0.518 & 0.518 & 0.0 & 0.507 & 0.507 & 0.0 \\
0.3 & 0.540 & 0.541 & 0.05 & 0.515 & 0.516 & 0.39 \\
0.4 & 0.571 & 0.572 & 0.18 & 0.527 & 0.529 & 0.57 \\
0.5 & 0.609 & 0.613 & 0.16 & 0.543 & 0.546 & 0.73 \\
0.6 & 0.658 & 0.661 & 0.45 & 0.563 & 0.567 & 0.71 \\
0.7 & 0.717 & 0.721 & 0.14 & 0.589 & 0.595 & 1.00 \\
0.8 & 0.785 & 0.795 & 0.25 & 0.623 & 0.630 & 1.1 \\
\hline
\end{tabular}

Table 3 Comparison of normalized stress-intensity factors, $F_{I}$ and $F_{I I}$, for embedded slant cracks in a rectangular plate subjected to remote uniform tensile stress. (Fig. 6(a), $(H / W)=2.0, \theta=75^{\circ}$ )

\begin{tabular}{cccccccc}
\hline & \multicolumn{3}{c}{$F_{I}=K_{I} /[S \sqrt{\pi a}]_{.}$} & & \multicolumn{3}{c}{$F_{I I}=K_{I} /[S \sqrt{\pi a}]$} \\
\cline { 2 - 4 }$(a / W)$ & $\begin{array}{c}\text { BEAM } \\
\text { value }\end{array}$ & $\begin{array}{c}\text { Reference } \\
\text { value }\end{array}$ & $\begin{array}{c}\text { Percent } \\
\text { difference }\end{array}$ & $\begin{array}{c}\text { BEAM } \\
\text { value }\end{array}$ & $\begin{array}{c}\text { Reference } \\
\text { value }\end{array}$ & $\begin{array}{c}\text { Percent } \\
\text { difference }\end{array}$ \\
\hline 0.1 & 0.068 & 0.068 & 0.0 & 0.252 & 0.252 & 0.0 \\
0.2 & 0.070 & 0.070 & 0.0 & 0.256 & 0.256 & 0.0 \\
0.3 & 0.074 & 0.074 & 0.0 & 0.263 & 0.263 & 0.0 \\
0.4 & 0.078 & 0.078 & 0.0 & 0.272 & 0.272 & 0.0 \\
0.5 & 0.083 & 0.084 & 1.2 & 0.282 & 0.283 & 0.35 \\
0.6 & 0.089 & 0.090 & 1.1 & 0.293 & 0.294 & 0.34 \\
0.7 & 0.094 & 0.096 & 2.1 & 0.305 & 0.306 & 0.33 \\
0.8 & 0.101 & 0.102 & 0.98 & 0.317 & 0.319 & 0.32 \\
\hline
\end{tabular}


Table 4 Comparison of normalized stress-intensity factors, $F_{I}$ and $F_{I I}$, for an embedded slant crack in a rectangular plate subjected to remote parabolic tensile stress. (Fig. 6(b), $(H / W)=2.0, \theta=45^{\circ}$ )

\begin{tabular}{cccccccl}
\hline & \multicolumn{3}{c}{$F_{I}=K_{I} /[S \sqrt{\pi a}]$} & & \multicolumn{3}{c}{$F_{I I}=K_{I I} /[S \sqrt{\pi a}]$} \\
\cline { 2 - 4 }$(a / W)$ & $\begin{array}{c}\text { BEAM } \\
\text { value }\end{array}$ & $\begin{array}{c}\text { Reference } \\
\text { value }\end{array}$ & $\begin{array}{c}\text { Percent } \\
\text { difference }\end{array}$ & $\begin{array}{c}\text { BEAM } \\
\text { value }\end{array}$ & $\begin{array}{c}\text { Reference } \\
\text { value }\end{array}$ & $\begin{array}{l}\text { Percent } \\
\text { difference }\end{array}$ \\
\hline 0.25 & 0.345 & 0.344 & 0.29 & & 0.361 & 0.363 & 0.55 \\
0.20 & 0.338 & - & - & 0.359 & - & - \\
0.30 & 0.353 & - & - & 0.365 & - & - \\
0.40 & 0.375 & - & - & 0.373 & - & - \\
0.50 & 0.403 & - & - & 0.384 & - & - \\
0.60 & 0.438 & - & - & 0.398 & - & - \\
0.70 & 0.479 & - & - & 0.415 & - & - \\
0.80 & 0.528 & - & - & 0.436 & - & - \\
\hline
\end{tabular}

Remote Parabolic Tensile Loading - Instead of remote uniform tensile loading, a parabolic tensile loading was applied to the slant crack specimens with a crack inclination angle of $45^{\circ}$ as shown in Fig. 6(b). The applied stresses $S(\xi)$ at a point on the top and bottom boundaries of the plate were calculated using the equation

$$
S(\xi)=S\left[1.0-\left(\frac{\xi}{W}\right)^{2}\right]
$$

where $-W \leq \xi \geq W$. The boundary element model used for this problem was same as the previous problem (see Fig. 7). The mode $I$ and mode $I I$ stressintensity factors are presented in Table 4 along with the reference value. Reference results are available only for a crack length of $(a / W)=0.25$ and again excellent agreement with the reference value was obtained with a very coarse boundary element idealization. A converged solution was obtained with three to five iterations.

These examples demonstrate that the BEAM gives accurate mixed-mode stress-intensity factors for problems of embedded cracks in plates with rectilinear boundaries. The method also effectively handled various loading conditions. 
In the next section the BEAM is applied to problems involving edge cracks in mixed-mode situations.

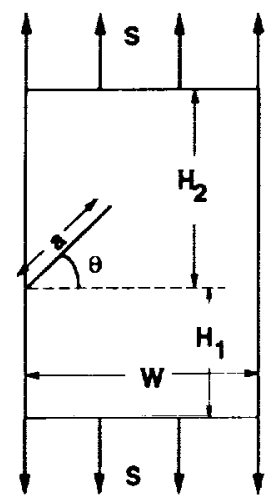

(a) Slant edge crackRemote tension loading

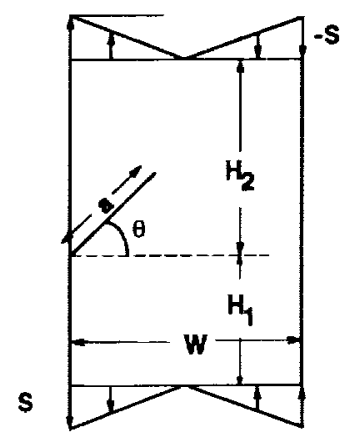

(b) Slant edge crackRemote bending loading

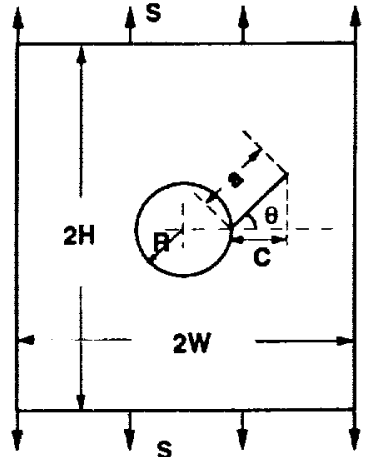

(c) Slant edge crack from \& circular hole

Figure 8. Slant edge crack problems analysed

\subsection{Slant Edge Cracks in Plates}

Remote Uniform Tensile Loading - A slant edge cracked plate with remote uniform uniaxial loading is shown in Fig. 8(a). A crack inclination angle of $45^{\circ}$ was used. The boundary element model with 56 nodes and 26 elements used in the analysis is shown in Fig. 9. Two finite elements were constructed near the point from which the crack is emanating as shown in Fig. 9. The distance $\Delta$ as discussed earlier was selected to be about 1.5 times the length of the smallest boundary-element near the edge. Crack lengths varying from $(a / W)=0.3$ to 0.6 were considered. The stress-intensity factors obtained using this model are presented in Table 5 along with the reference solutions. The maximum difference between the results from this analysis and the reference solution is about 3 percent. About 10 to 12 iterations were needed to obtain converged solutions.

Even though the shift of the origin is not necessary for the edge cracks emanating from external boundaries, the origin-shifting method is used in the above analysis, since numerical experimentation showed that the origin shift gave better 
results than the one without origin shifting. This may be due to the effect of the fictitious crack tip is minimized in the origin shifting method. In the above analysis the fictitious crack tip was positioned at a distance $(x / W)=-0.3$ from the free edge.

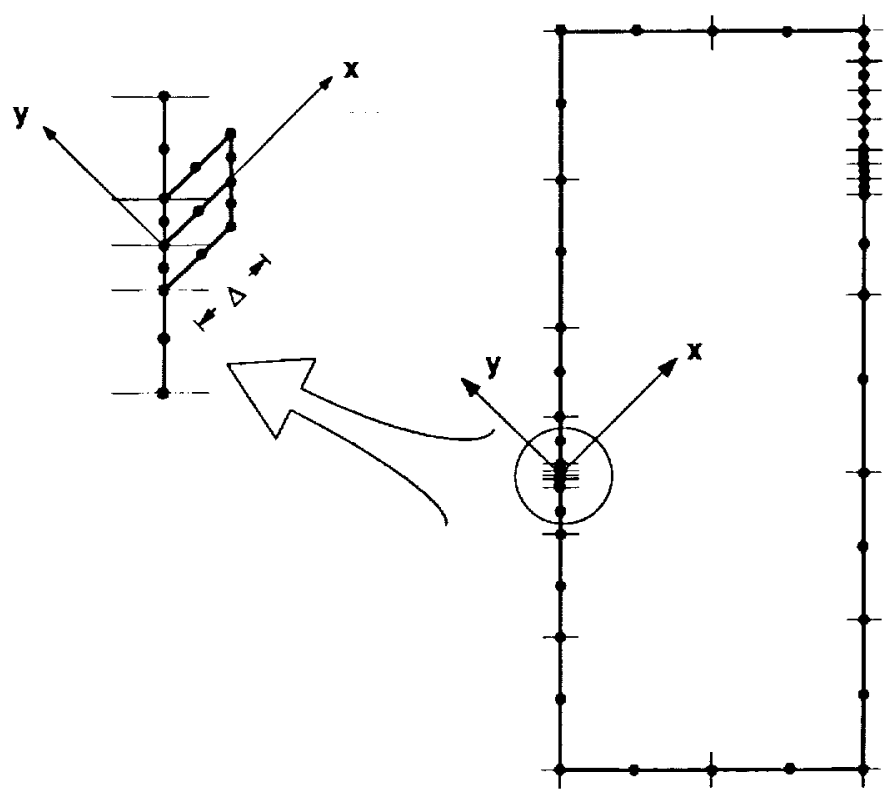

Figure 9. Boundary element model for edge crack problems

Remote Bending Loading - A slant edge cracked plate with remote bending is shown in Fig. 8(b). The boundary element model is shown in Fig. 9. The bending moment was simulated by the corresponding bending stress applied over the edges as shown in Fig. 8(b). Crack lengths ranging from $(a / W)=0.3$ to 0.6 were considered. The results are presented in Table 6 with reference results from the litreature. Here again excellent agreement was obtained with the reference solutions. 
Table 5 Comparison of normalized stress-intensity factors, $F_{I}$ and $F_{I I}$, for slant edge cracks in a rectangular plate subjected to remote uniform tensile stress with origin shift. (Fig. $8(\mathrm{a}),\left(H_{1} / W\right)=1.0,\left(H_{2} / W\right)=1.5, \theta=45^{\circ}$ )

\begin{tabular}{|c|c|c|c|c|c|c|}
\hline \multirow[b]{2}{*}{$(a / W)$} & \multicolumn{3}{|c|}{$F_{I}=K_{I} /[S \sqrt{\pi a}]$} & \multicolumn{3}{|c|}{$F_{I I}=K_{I I} /[S \sqrt{\pi a}]$} \\
\hline & $\begin{array}{c}\text { BEAM } \\
\text { value }\end{array}$ & $\begin{array}{l}\text { Reference } \\
\text { value }\end{array}$ & $\begin{array}{c}\text { Percent } \\
\text { difference }\end{array}$ & $\begin{array}{c}\text { BEAM } \\
\text { value }\end{array}$ & $\begin{array}{c}\text { Reference } \\
\text { value }\end{array}$ & $\begin{array}{l}\text { Percent } \\
\text { difference }\end{array}$ \\
\hline 0.10 & 0.728 & - & - & 0.400 & - & - \\
\hline 0.20 & 0.792 & - & - & 0.425 & - & - \\
\hline 0.30 & 0.889 & 0.879 & 1.1 & 0.465 & 0.450 & 3.3 \\
\hline 0.35 & 0.943 & 0.940 & 0.32 & 0.490 & 0.473 & 3.6 \\
\hline 0.40 & 1.009 & 1.011 & 0.20 & 0.518 & 0.505 & 2.6 \\
\hline 0.45 & 1.080 & 1.094 & 1.3 & 0.551 & 0.536 & 2.8 \\
\hline 0.50 & 1.184 & 1.190 & 0.50 & 0.590 & 0.574 & 2.8 \\
\hline 0.55 & 1.311 & 1.301 & 0.77 & 0.636 & 0.616 & 3.3 \\
\hline 0.60 & 1.434 & 1.437 & 1.2 & 0.699 & 0.674 & 3.7 \\
\hline
\end{tabular}

Table 6 Comparison of normalized stress-intensity factors, $F_{I}$ and $F_{I I}$, for slant edge cracks in a rectangular plate subjected to remote bending stress. (Fig. 8(b), $\left.\left(H_{1} / W\right)=1.0,\left(H_{2} / W\right)=1.5, \theta=45^{\circ}\right)$

\begin{tabular}{|c|c|c|c|c|c|c|}
\hline \multirow[b]{2}{*}{$(a / W)$} & \multicolumn{3}{|c|}{$F_{I}=K_{I} /[S \sqrt{\pi a}]$} & \multicolumn{3}{|c|}{$F_{I I}=K_{I I} /[S \sqrt{\pi a}]$} \\
\hline & $\begin{array}{c}\text { BEAM } \\
\text { value }\end{array}$ & $\begin{array}{c}\text { Reference } \\
\text { value }\end{array}$ & $\begin{array}{c}\text { Percent } \\
\text { difference }\end{array}$ & $\begin{array}{c}\text { BEAM } \\
\text { value }\end{array}$ & $\begin{array}{l}\text { Reference } \\
\text { value }\end{array}$ & $\begin{array}{l}\text { Percent } \\
\text { difference }\end{array}$ \\
\hline 0.10 & 0.674 & - & - & 0.360 & - & - \\
\hline 0.20 & 0.673 & - & - & 0.345 & - & - \\
\hline 0.30 & 0.690 & 0.685 & 0.73 & 0.334 & 0.315 & 6.0 \\
\hline 0.35 & 0.705 & 0.705 & 0.0 & 0.332 & 0.319 & 4.1 \\
\hline 0.40 & 0.728 & 0.736 & 1.1 & 0.330 & 0.320 & 3.1 \\
\hline 0.45 & 0.756 & 0.758 & 0.26 & 0.331 & 0.324 & 2.2 \\
\hline 0.50 & 0.789 & 0.795 & 0.76 & 0.340 & 0.329 & 3.3 \\
\hline 0.55 & 0.827 & 0.832 & 0.6 & 0.341 & 0.337 & 1.2 \\
\hline 0.60 & 0.872 & 0.889 & 1.9 & 0.352 & 0.344 & 2.3 \\
\hline
\end{tabular}




\subsection{Slant Edge Crack Emanating From A Hole In A Rectangular Plate}

An edge crack emanating from a hole with radius $R$ in an infinite rectangular plate and loaded with remote uniform tension is shown in Fig. 8(c). The crack inclination angle $\theta$ was selected as $30^{\circ}$. Since the reference solution available for this problem is of an infinite plate, a plate with dimensions $(H / W)=1.0$ and $(W / R)=24$ was considered. The outer boundaries of the plate were modeled with 12 elements as shown in Fig. 10 (a). The hole boundary was modeled with 78 boundary elements as shown in Fig. 10(b). (Note that the middle node of the boundary elements are not shown in Fig. 10(b).) Here again two finite elements were constructed near the crack mouth as shown in Fig. 10(c). As described in [12], for all crack lengths with $(a / R)>0.25$, the fictitious crack tip was positioned at $(x / R)=-0.25$. The crack lengths ranged from $(c / R)=0.1$ to 1.0 . (Note that $c$ is the projected length of the crack along the horizontal, see Fig. 8(c).). The stress-intensity factors obtained with this method are presented in Table 7 along with the reference solution. Excellent agreement was obtained with the reference solutions. Only 10 to 14 iterations were needed to obtain converged solutions.

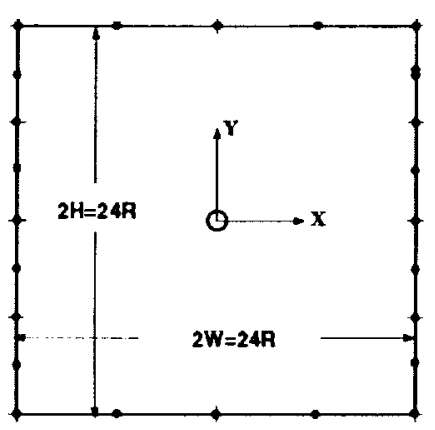

(a) Model for the plate

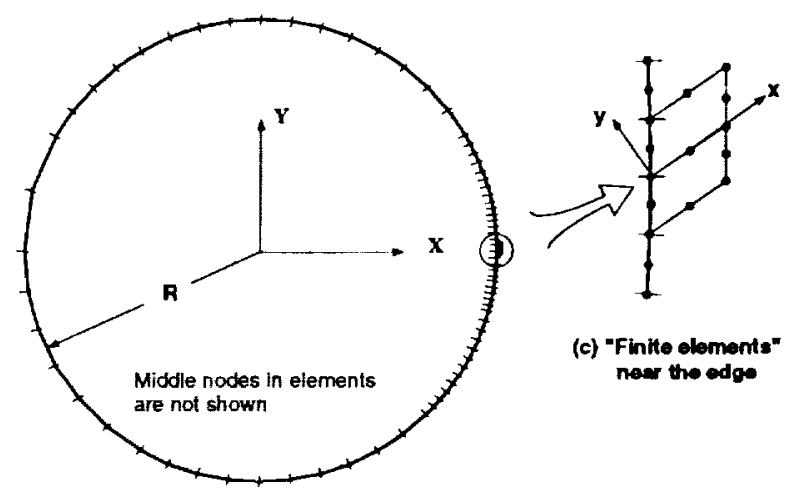

(b) Model for the circular hole

Figure 10. Boundary element model for an edge crack from a circular hole problem 
Table 7 Comparison of normalized stress-intensity factors, $F_{I}$ and $F_{I I}$, for slant edge cracks emanating from a circular hole in a rectangular plate subjected to remote uniform tensile stress. (Fig. $8(\mathrm{c}),(H / W)=1.0, \theta=30^{\circ}$ )

\begin{tabular}{|c|c|c|c|c|c|c|}
\hline \multirow[b]{2}{*}{$(c / R)$} & \multicolumn{3}{|c|}{$F_{I}=K_{I} /[S \sqrt{\pi c}]$} & \multicolumn{3}{|c|}{$F_{I I}=K_{I I} /[S \sqrt{\pi c}]$} \\
\hline & $\begin{array}{c}\text { BEAM } \\
\text { value }\end{array}$ & $\begin{array}{l}\text { Reference } \\
\text { value }\end{array}$ & $\begin{array}{c}\text { Percent } \\
\text { difference }\end{array}$ & $\begin{array}{c}\text { BEAM } \\
\text { value }\end{array}$ & $\begin{array}{c}\text { Reference } \\
\text { value }\end{array}$ & $\begin{array}{c}\text { Percent } \\
\text { difference }\end{array}$ \\
\hline 0.10 & 2.501 & 2.501 & 0.0 & 0.750 & 0.701 & 7.0 \\
\hline 0.15 & 2.328 & $(2.300)^{*}$ & 1.2 & 0.677 & $(0.660)$ & 2.6 \\
\hline 0.20 & 2.165 & 2.140 & 1.2 & 0.617 & 0.610 & 1.2 \\
\hline 0.30 & 1.866 & $(1.880)$ & 0.75 & 0.548 & $(0.550)$ & 0.36 \\
\hline 0.40 & 1.645 & $(1.680)$ & 2.1 & 0.519 & $(0.520)$ & 0.19 \\
\hline 0.50 & 1.501 & 1.519 & 1.2 & 0.506 & 0.511 & 0.98 \\
\hline 0.75 & 1.226 & $(1.240)$ & 1.1 & 0.490 & $(0.500)$ & 2.0 \\
\hline 1.00 & 1.060 & 1.090 & 2.8 & 0.491 & 0.508 & 3.3 \\
\hline
\end{tabular}

* Values in parantheses are interpolated values from tables in reference 4 (pages 259 and 261).

\section{Concluding remarks}

A boundary element alternating method (BEAM) for two-dimensional, mixed-mode fracture problems is presented. The analytical solution for arbitrary normal and tangential pressure distributions on the faces of the crack in an infinite plate is used as the fundamental solution. In the numerical part, the boundary element method is used to model and analyze the uncracked body. Details of the implementation of the algorithm, together with a variety of computational aspects of the method, were presented. An origin-shifting method is presented that is particularly useful in the analysis of edge cracks emanating from the internal boundaries. This method of origin-shifting was also found to give better results even for edge cracks emanating from internal boundaries. Also for edge cracks problems, a procedure which utilizes "finite elements" and finite-element type interpolations and stress evaluation was developed to calculate the stresses accurately near a boundary. This procedure yielded stresses that are of better accuracy than the stresses calculated by the traditional boundary element method. 
The BEAM was applied to several mixed-mode problems to evaluate its effectiveness. These numerical examples showed that the BEAM requires very little modeling effort and yields accurate mixed-mode stress-intensity factors. Three to four iterations were necessary to yield accurate and converged stress-intensity factors, while edge crack configurations needed about 10-15 iterations. With this method, several crack lengths can be analyzed in a single computer run and hence it can be used to economically obtain stress-intensity factors over a range of cracklengths.

\section{Acknowledgements}

This work was performed, in part, at the Computational Mechanics Branch, Structural Mechanics Division, NASA Langley Research Center as a part of NASA Contract NAS1-19317. 


\section{REFERENCES}

[1] Tada, H., Paris, P. C., and Irwin, G.R., The Stress Analysis of Cracks Handbook, Del Research Corporation, Hellertown, Pa., 1973.

[2] Rooke, D. P., and Cartwright, D. J., Compendium of Stress Intensity Factors, The Hillingdon Press, Uxbridge, Middlesex, 1976.

[3] Sih, G. C., Handbook of Stress Intensity Factors, Volumes 1 \& 2, Lehigh University, Bethelhem, Pa., 1973.

[4] Murakami, Y., et al (Eds), Stress Intensity Factors Handbook, Volumes 1 \& 2, Pergamon Press, 1987.

[5] Kantorovich, L. V., and Krylov, V. I., Approximate Methods of Higher Analysis, Interscience, N.Y, 1964.

[6] Nishioka, T., and Atluri, S. N., "Analytical Solution for Embedded Elliptical Cracks and Finite Element-Alternating Method for Elliptical Surface Cracks, Subjected to Arbitrary Loadings," Engineering Fracture Mechanics, Vol. 17, 1983, pp. 247-268.

[7] Nishioka, T., and Atluri, S. N., "An Alternating Method for Analysis of Surface Flawed Aircraft Structural Components," AIAA Jnl., Vol. 21, 1983, pp. 749-757.

[8] Atluri, S. N., and Nishioka, T., "Computational Methods for ThreeDimensional Problems of Fracture," Chapter 7, In Computational Methods in Mechanics of Fracture, S. N. Atluri (Ed), North Holland, 1986, pp. 230-287.

[9] Raju, I. S., Atluri, S. N., and Newman, J. C., Jr., "Stress-Intensity Factors for Small Surface and Corner Cracks in Plates," Paper Presented at the 20th National Symposium on Fracture Mechanics, Lehigh University, Bethlehem, Pa, June 23-25, 1987.

[10] Raju, I. S., and Fichter, W. B., "A Finite-Element Alternating Method for Two-Dimensional mode $I$ Crack Configurations," Engineering Fracture Mechanics, Vol. 33, 1989, pp. 525-540.

[11] Chen, K-L., and Atluri, S. N., "A Finite-Element Alternating Method for Cost-Effective Determination of Weight-Function and S. I. F.'s for 
Mutliple Cracks in Mixed-Mode Fracture," in Computational Mechanics '88, Theory and Applications, S. N. Atluri and G. Yagawa (Eds), SpringerVerlag, Vol. 1, 1988, pp. 11.ii.1-11.ii-6.

[12] Krishnamurthy, T., and Raju, I. S., "A Finite-Element Alternating Me thod for Two-Dimensional Mixed-Mode Crack Configurations", Engineering Fracture Mechanics, Vol. 36, 1990, pp. 297-311.

[13] Rajiah, H., and Atluri, S. N., "Evaluation of K-factors and Weight Functions for 2-D Mixed-mode Multiple Cracks by the Boundary Element Alternating Method", in Computational Mechanics '88, Theory and Applications, S. N. Atluri and G. Yagawa (Eds), Springer-Verlag, Vol. 1, 1988, pp. 11.i.1-11.i-5.

[14] Brebbia, C. A., and Dominguez, J., Boundary Elements- An Introductory Course, Coputational Mechanics Publications and McGraw-Hill, New York, 1989.

[15] Hinton, E., and Campbell, J. S., "Local and Global Smoothing of Discontinuous Finite-Element Functions Using Least Squares Method," Int. Jnl. Num. Meth. Engng., Vol. 8, 1974, pp. 461-480.

[16] Hinton. E., and Campbell, J. S., "Local Least Square Stress Smoothing for Parabolic Isoparametric Elements," Int. Jnl. Num. Meth. Engng., Vol. 9, 1975, pp. 235-256. 


\section{Appendix - A}

Global-local Transformations in the BEAM

This appendix describes the coordinate and stress transformations that are required when the global coordinate system does not coincide with the local (crack) coordinate system.

The analytical part of the alternating method assumes that the crack is along the $y=0$ line (i.e. $x$ - axis). While the crack coordinate, $(x-y)$, system is convenient for the analytical solution, it is cumbersome for the boundary element part of the method. For example in Fig. A-1 the global, $(X-Y)$, coordinate system is obviously convenient for the BE part of the method and not the $x-y$ crack coordinates system. These two coordinate systems require stress and coordinate transformations in the BEAM. These are discussed below.

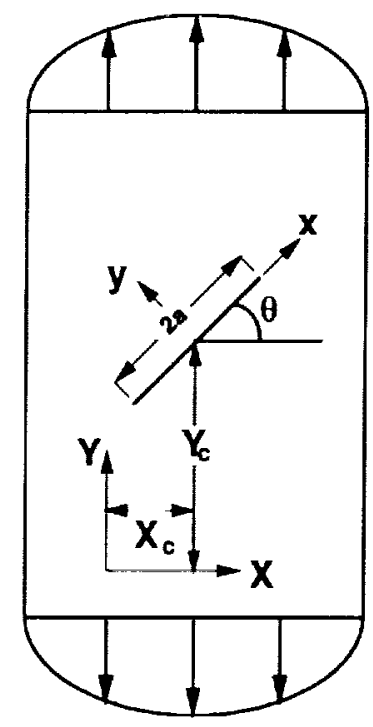

Figure A-1, Global-local coordinate syslems in BEAM

Before performing the Step 2 of the BEAM, the stresses, $\sigma_{X}, \sigma_{Y}$ and $\sigma_{X Y}$, obtained from the uncracked body solution need to transformed to the local (crack) coordinate system as, 


$$
\left\{\begin{array}{c}
\sigma_{x} \\
\sigma_{y} \\
\sigma_{x y}
\end{array}\right\}=[\lambda]\left\{\begin{array}{c}
\sigma_{X} \\
\sigma_{Y} \\
\sigma_{X Y}
\end{array}\right\}
$$

In Eq. $(A-1),[\lambda]$ is the transformation matrix given by

$$
[\lambda]=\left[\begin{array}{ccc}
\cos ^{2} \theta & \sin ^{2} \theta & \sin 2 \theta \\
\sin ^{2} \theta & \cos ^{2} \theta & -\sin 2 \theta \\
\sin \theta \cos \theta & \sin \theta \cos \theta & \cos 2 \theta
\end{array}\right]
$$

where $\theta$ is the crack inclination angle measured from the $X$-axis of the global coordinate system as shown in Fig. A-1. The transformed stresses $\sigma_{x}, \sigma_{y}$ and $\sigma_{x y}$ are then used to obtain the normal and tangential tractions on the crack faces.

Step 6 of the alternating method, on the other hand, requires an inverse transformation. Recall that in Step 6 of the method the stresses on all the boundaries of the body due to crack face pressures $p_{x}^{R}$ and $p_{y}^{R}$ are calculated. From these stresses the tractions on the boundaries are calculated. First to calculate the stresses at a point $\left(X_{b}, Y_{b}\right)$ on the boundary, the coordinates of this point in the local system are calculated as

$$
\left\{\begin{array}{l}
x_{b} \\
y_{b}
\end{array}\right\}=\left[\begin{array}{cc}
\cos \theta & -\sin \theta \\
\sin \theta & \cos \theta
\end{array}\right]\left\{\begin{array}{c}
X_{b}-X_{c} \\
Y_{b}-Y_{c}
\end{array}\right\}
$$

where $X_{c}, Y_{c}$ are the global coordinates of origin of the local coordinate system. Then the complex variable at this point can be formed as, $z_{b}=x_{b}+i y_{b}$. This complex variable is then used in Eq. (22) to evaluate stresses in the local coordinate system. These stresses are then transformed to the global system using

$$
\left\{\begin{array}{c}
\sigma_{X} \\
\sigma_{Y} \\
\sigma_{X Y}
\end{array}\right\}=[\lambda]^{-1}\left\{\begin{array}{c}
\sigma_{x} \\
\sigma_{y} \\
\sigma_{x y}
\end{array}\right\}
$$

These global stresses are then used in Eq. (24) in Step 6.

With the above definitions, however, Eq. (25) need to be redefined as, 


$$
\begin{aligned}
& \{T\}^{T}=\left\{\begin{array}{ll}
T_{X} & T_{Y}
\end{array}\right\} \\
& {[q]=\left[\begin{array}{ccc}
n_{X} & n_{Y} & 0 \\
0 & n_{Y} & n_{X}
\end{array}\right]}
\end{aligned}
$$

where $n_{X}$ and $n_{Y}$ are the direction cosines of the normal to the boundary with respect to $X$ - and $Y$ - axes, respectively. 


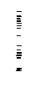
$=$ 


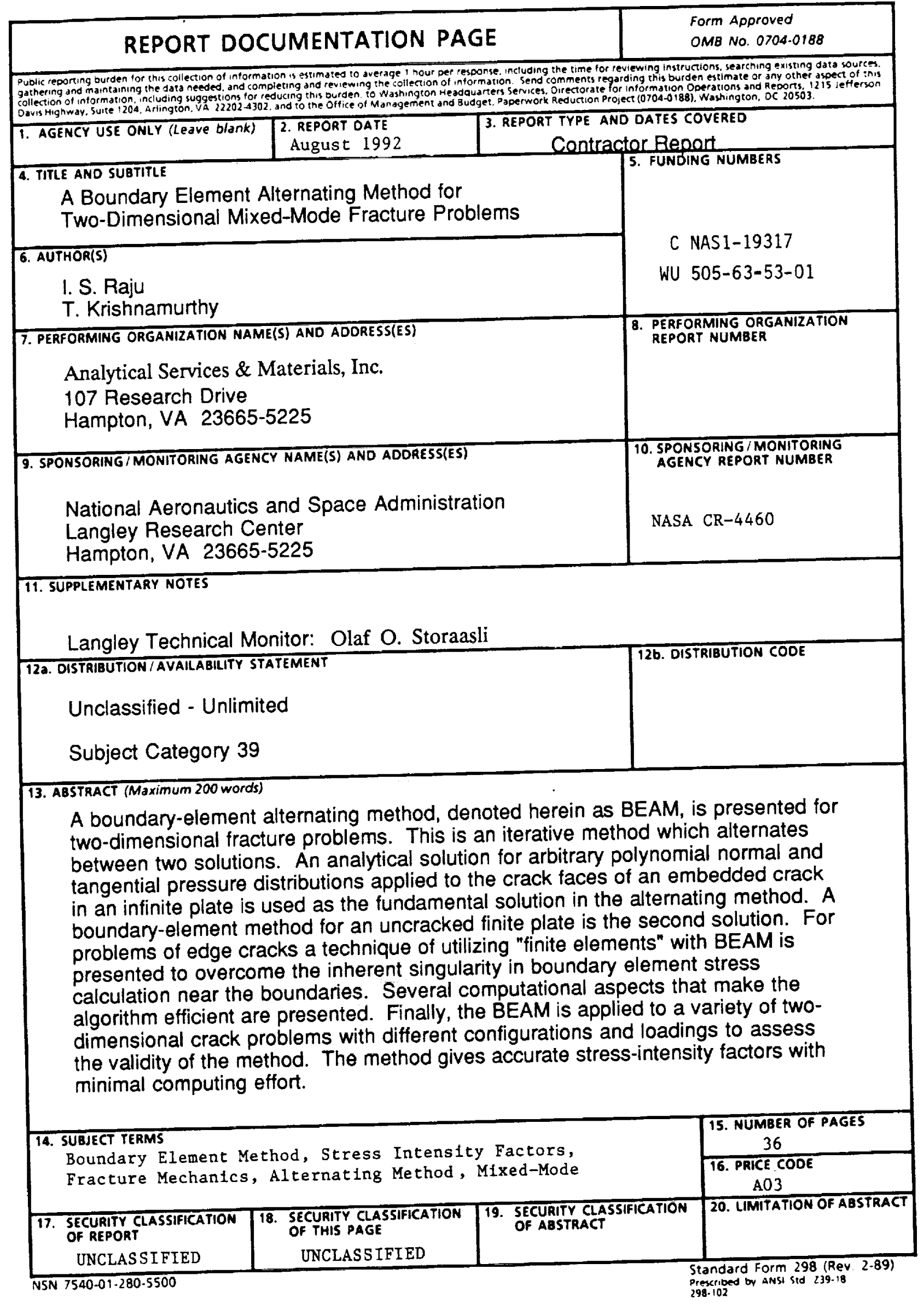


